01

\title{
Ридберговские состояния радикала ОН
}

\author{
(C) А.С. Корнев ${ }^{1}$, В.Е. Чернов ${ }^{1, *}$, Б.А. Зон ${ }^{1}$, Д.Л. Дорофреeв ${ }^{1}$, P. Kubelik ${ }^{2}$, M. Ferus ${ }^{2}$ \\ ${ }^{1}$ Воронежский государственный университет, \\ 394018 Воронеж, Россия \\ ${ }^{2} \mathrm{~J}$. Heyrovský Institute of Physical Chemistry, Academy of Sciences of the Czech Republic, \\ 18223 Prague, Czech Republic \\ * e-mail: chernov@niif.vsu.ru
}

Поступила в редакцию 21.06.2021 г.

В окончательной редакции 21.06.2021 г.

Принята к публикации 06.08.2021 г.

Рассмотрены ридберговские состояния радикала ОН в адиабатическом (вращательное приближение Борна-Оппенгеймера) и в обратном ему пределах. Необходимое значение $d=0.833$ дипольного момента катиона $\mathrm{OH}^{+}$вычислено методом RCCSD(T)/aug-cc-pV5Z. Результаты расчетов показывают, что такой дипольный момент оказывает относительно малое влияние на энергии ридберговских состояний. Исключение составляют состояния, порождаемые $s$-состояниями в центрально-симметричном поле, для которых влияние дипольного момента оказывается весьма значительным. В обратном вращательном приближении Борна-Оппенгеймера подробно рассмотрена зависимость ридберговского спектра от полного углового момента молекулы $J$. Эта зависимость принципиально отличается от хорошо известной зависимости от полного момента энергий вращающегося волчка $\sim J(J+1)$.

Ключевые слова: полярные молекулы, мультпольные моменты, поляризуемость, гидроксил.

DOI: $10.21883 /$ OS.2021.11.51634.2462-21

\section{Введение}

Процессы с участием высоковозбужденных (ридберговских) состояний атомов и молекул важны во многих разделах физики и химии. Сказанное касается и радикала $\mathrm{OH}$, присутствующего в заметных количествах в атмосфере Земли и других планет. Гидроксильный радикал образуется в атмосфере в результате фотолиза [1] или диссоциативного возбуждения молекул воды свободными электронами [2]. Отметим, что в газоразрядной плазме возможен дополнительный механизм образования гидроксильного радикала за счет передачи энергии от метастабильных атомов аргона молекулам воды [3]. Радикал ОН способствует образованию окислов азота из атмосферного азота [4]. Облака ОН занимают значительные объемы во Вселенной, поэтому наблюдение ридберговских спектров ОН может дать полезную информацию о физических условиях в этих объектах. Суточные колебания поглощения ОН в лунной атмосфере в ближнем ИК диапазоне могут дать важную информацию о формировании молекул воды в результате поглощения протонов солнечного ветра [5], падения метеоритов или комет с ледяным ядром [5], либо в результате фотолиза [7-9]. Кроме того, этот радикал рассматривается как один из кандидатов для определения изменения со временем мировых постоянных (см., например, [10-15]).

Одна из основных проблем, связанных с нахождением ридберговских спектров полярных молекул, обусловлена перемешиванием дипольным моментом молекулярного остова состояний ридберговского электрона (RE) с разными орбитальными моментами. Известно несколько вариантов решения этой проблемы, например, многоканальная теория квантового дефекта (MQDT), предложенная Ситоном [16,17]. Эта теория развивалась многими авторами в первую очередь для неполярных молекул [18-22]. Позже MQDT применялась и к некоторым полярным молекулам, в частности, NO [23], $\mathrm{CaF}$ [24-29], $\mathrm{BaF}$ [15,30-33], $\mathrm{CaCl}$ [34-36], SO [37] и др.

MQDT применим в области больших квантовых чисел $\mathrm{RE}, n \gg 1$, и обладает в этой области относительно высокой точностью. Однако в некоторых случаях могут быть применены более простые в вычислительном отношении подходы. Один из таких подходов основан на приближенном представлении потенциала молекулярного остова в виде суперпозиции потенциалов точечного заряда и точечного диполя. В рамках этого подхода в двух предельных случаях были получены аналитические решения уравнения Шредингера для RE.

Одним из этих предельных случаев является адиабатическое приближение, известное также как приближение Борна-Оппенгеймера (BОА), когда вращение остова происходит значительно медленнее, чем движение RE. Соответствующее аналитическое решение было дано в работах [38,39]. Именно в работах $[38,39]$ из состояний RE с целочисленными орбитальными моментами были построены состояния с некоторыми эффективными нецелочисленными, в общем случае, орбитальными моментами, которые учитывают влияние перемешивания состояний дипольным моментом остова. 
Вторым предельным случаем является обратное приближение Борна-Оппенгеймера (IBOA), когда остов считается быстро вращающимся по сравнению с движением RE. Такой предельный случай возможен, если главное квантовое число RE достаточно велико. Аналитическое решение уравнения Шредингера для IBOA получено в работе [40]. При переходе от ВОА к IBOA происходит радикальная перестройка спектра RE, в частности, его зависимость от дипольного момента остова. Подчеркнем, что IBOA в нашем случае связано с медленностью движения, соответствующего электронным степеням свободы, по сравнению с вращательными, а не с колебательными, как это обычно понимается для низковозбужденных состояний молекул [41-43].

Расчеты ридберговских спектров молекулы SO в предельных случаях ВОА и IBОА изложены в работе [44]. В данной работе аналогичные расчеты проводятся для радикала ОН.

C ростом главного квантового числа RE порождаемые квантовым дефектом смещения уровней уменьшаются по абсолютной величине. Когда они становятся сравнимыми с расщеплением остовного $\Lambda$-дублета, в ридберговском спектре полярной молекулы могут наблюдаться аномалии, обсуждавшиеся в общем случае в работе [45], а для молекулы SO в работе [44]. В данной работе эти аномалии не рассматриваются. Отметим лишь, что именно переход между уровнями $\Lambda$-дублета в радикале $\mathrm{OH}$ (длина волны $\simeq 18 \mathrm{~cm}$ ) анализировался в работах [10-15].

Также в данной работе не рассматриваются кривые потенциальной энергии для молекулы ОН и ее колебательные спектры. Соответственно не анализируются и возмущения спектра, связанные с предиссоциацией. Предиссоциация $A^{2} \Sigma^{+}$-состояния радикала $\mathrm{OH}$ исследовалась теоретически и экспериментально в ряде работ (см., например, [46] и ссылки в ней), однако предиссоциация более высоких ридберговских состояний, являющихся предметом данной работы, требует особого рассмотрения.

В работе, где не оговорено особо, используется атомная система единиц.

\section{Основные уравнения}

\section{Адиабатический предел (BОА)}

Для учета перемешивания состояний RE c разными орбитальными моментами $l$, дипольным моментом $d$ в работах $[38,39,47]$ вместо обычных сферических функций $Y_{l m}(\theta, \varphi)$ вводятся угловые функции

$$
Z_{\mathrm{BOA}}(d ; \theta, \varphi)=\sum_{l=|m|}^{\infty} a_{l} Y_{l m}(\theta, \varphi),
$$

где $m$ - проекция орбитального момента RE на направление $z$ дипольного момента молекулярного остова, а коэффициенты $a_{l}$ являются собственными векторами линейной трехдиагональной системы однородных алгебраических уравнений, соответствующих собственным числам $\mathscr{L}$ :

$$
\begin{aligned}
& l(l+1) a_{l}+2 d \sum_{l^{\prime}=l \pm 1}\left(\frac{2 l^{\prime}+1}{2 l+1}\right)^{1 / 2} \\
& \times C_{l^{\prime} 010}^{l 0} C_{l^{\prime} m 10}^{l m} a_{l^{\prime}}=\mathscr{L} a_{l}, \quad|m| \leq l<\infty .
\end{aligned}
$$

Собственные числа $\mathscr{L}$ и собственные векторы системы уравнений (2) будем нумеровать целыми числами $L$, изменяющимися в тех же пределах, что и индекс суммирования $l$ в (2), а именно $|m| \leq L<\infty$. Причем нумерацию выберем так, что при $d \rightarrow 0$

$$
\mathscr{L}^{(L)} \rightarrow L(L+1), \quad a_{l}^{(L)} \rightarrow \delta_{L l} .
$$

В общем случае удобно ввести эффективные орбитальные моменты $\tilde{L}$, определяемые соотношением

$$
\mathscr{L}^{(L)}=\tilde{L}(\tilde{L}+1), \quad \tilde{L}=\sqrt{\mathscr{L}^{(L)}+1 / 4}-1 / 2 .
$$

Спектр RE выражается через $\tilde{L}$ соотношением

$$
\varepsilon_{v L}=-1 / 2 v^{2}, \quad v=n_{r}+\tilde{L}+1 .
$$

Здесь $n_{r}=0,1, \ldots$ есть радиальное квантовое число. Отметим, что при $\mathscr{L}^{(L)}<-1 / 4$, что возможно при достаточно большой величине дипольного момента, спектр RE, а вместе с ним и спектр всей молекулы становится комплексным. Это означает, как известно, неустойчивость системы, а именно падение RE в центр молекулярной системы координат [48]. Применительно к рассматриваемой здесь задаче возникновение такого поведения свидетельствует о неприменимости приближения точечного остова для состояний с $\mathscr{L}^{(L)}<-1 / 4$. Более подробно этот вопрос рассматривается, например, в работе [49].

Вклад квадрупольного момента и поляризуемости остова в квантовый дефект в первом приближении теории возмущений:

$$
\begin{gathered}
\mu_{Q}=\frac{2\left(L(L+1)-3 m^{2}\right)}{L(L+1)(2 L-1)(2 L+1)(2 L+3)} Q_{z z}, \\
\mu_{\mathrm{pol}}=\frac{12\left(1-L(L+1) / 3 n^{2}\right)}{L(L+1)(2 L-1)(2 L+1)(2 L+3)} \\
\times\left(\frac{\bar{\alpha}}{2}+\frac{\gamma}{3} \frac{L(L+1)-3 m^{2}}{(2 L+1)(2 L+3)}\right) \\
\bar{\alpha}=\frac{2 \alpha_{\perp}+\alpha_{\|}}{3}, \quad \gamma=\alpha_{\|}-\alpha_{\perp}, \quad n=n_{r}+L+1 .
\end{gathered}
$$

Приведенные выражения для $\mu_{Q}$ и $\mu_{\text {pol }}$ справедливы при $L \neq 0$. При $L=0$ имеем $\mu_{Q}=0$, а соответствующее выражение для $\mu_{\text {pol }}$ оказывается расходящимся, что также говорит о неприменимости в этом случае приближения точечного остова. 


\section{Обратное вращательное приближение Борна-Оппенгеймера (IBOA)}

Решение уравнения Шредингера для электрона, движущегося в кулоновском поле и поле быстро вращающегося точечного диполя, найдено в работе [40] для симметричного волчка. Для двухатомной молекулы, рассматриваемой в данной работе, общие формулы несколько упрощаются.

Согласно [40] волновая функция молекулы в IBOA ищется в виде:

$$
\Psi_{J M}^{j \lambda}=\sqrt{\frac{2 j+1}{8 \pi^{2}}} R(r) \sum_{l j_{z} m} C_{j j_{z} l m}^{J M} D_{\omega j_{z}}^{j}(\Omega) Y_{l m}(\mathbf{r} / r) a_{l}^{\varkappa} .
$$

Здесь индекс $\varkappa$ обозначает набор индексов $(J, j, \omega)$, $J$ - полный момент молекулы, $M$ - его проекция на ось $z$ лабораторной системы координат (ЛСК), $j, j_{z}-$ полный момент остова и его проекция на ось $z$ ЛСК соответственно, $\omega$ - проекция момента остова на ось молекулы, совпадающей с направлением дипольного момента, $D-$ матрица конечных вращений, зависящая от углов Эйлера остова $\Omega, R(r)$ - радиальная волновая функция RE.

Решение уравнения Шредингера, полученное в [40], сводится к нахождению собственных чисел $\mathscr{L}_{\varkappa}$ и собственных векторов $\left\{a_{l}^{\chi}\right\}$ теперь уже в отличие от $[38,39]$ конечной системы линейных алгебраических уравнений, определяющих угловую функцию

$Z_{\mathrm{IBOA}}(d ; \theta, \varphi)$, аналогичной $(2)$ :

$$
\begin{gathered}
l(l+1) a_{l}^{\varkappa}+2(-1)^{2 j} d \omega\left[\frac{(2 j+1)(2 l+1)}{j(j+1)}\right]^{1 / 2} \\
\times \sum_{l^{\prime}=l \pm 1} C_{l 010}^{l^{\prime} 0} W\left(j 1 J l^{\prime} ; j l\right) a_{l^{\prime}}^{\varkappa}=\mathscr{L}_{\varkappa} a_{l}^{\varkappa}, \\
|J-j| \leq l \leq J+j,
\end{gathered}
$$

где $W$ - коэффициенты Рака. Зависимость собственных чисел $\mathscr{L}_{x}$ от $J$, следующая из этой формулы, определяет зависимость энергии молекулы от ее полного момента. Поскольку мы не учитываем спин RE, из свойств коэффициента Клебша-Гордана, входящего в формулу (8), следует, что полный момент молекулы $J$ является целым или полуцелым в зависимости от того, является ли целым или полуцелым момент остова $j$.

Собственные числа и собственные векторы системы уравнений (9) будем также нумеровать целыми числами $L$, которые теперь изменяются в пределах $|J-j| \leq L \leq J+j$. По аналогии с формулами (3), (5)

$$
\begin{gathered}
\mathscr{L}_{\varkappa}^{(L)} \rightarrow L(L+1), \quad a_{l}^{\varkappa(L)} \rightarrow \delta_{L l}, \quad \text { при } \quad d \rightarrow 0, \\
\varepsilon_{\varkappa v L}=-1 / 2 v^{2}, \quad v=n_{r}+\mathscr{L}+1, \quad n_{r}=0,1, \ldots
\end{gathered}
$$

Заметим, что при $\omega=0$, что возможно при целых $j$, из уравнений (9) следует $\mathscr{L}_{x}=l(l+1)$, что лишь обозначениями отличается от предельных значений (10).
Иными словами, при $\omega=0$ дипольный момент не влияет на движение RE. Физический смысл этого факта совершенно понятен: при $\omega=0$ дипольный момент остова перпендикулярен оси его вращения, и величина дипольного момента при быстром вращении остова усредняется.

Полная энергия молекулы определяется выражением

$$
E_{\varkappa v L}=B j(j+1)+\varepsilon_{\varkappa v L},
$$

где $B-$ вращательная постоянная остова. Поскольку $\varepsilon_{\varkappa v L}<0$, из формулы (11) следует, что ниже каждого вращательного уровня остова с моментом $j$ располагается кулоноподобный спектр RE, расщепленный по $L$ и $J$. Уравнения (2), (9) являются основными. После их решения ридберговские спектры находятся по формулам (5), (10). Более сложные случаи связи внутримолекулярных угловых моментов рассмотрены в [50].

\section{Дипольный и квадрупольный моменты и поляризуемость радикал-катиона $\mathrm{OH}^{+}$}

Для решения уравнений (2), (9) необходимо знание дипольного момента радикала $\mathrm{OH}^{+}$, а также его квадрупольного момента и поляризуемости (для оценки квантовых дефектов по формулам (6) и (7)). Мы использовали две группы методов для расчета мультипольных моментов.

В первом случае расчет проводился с помощью современных программ квантово-химических расчетов: Gaussian [51], MOLPRO [52] и NWChem [53]. Были задействованы такие методы, как теория функционала плотности (DFT), Møller-Plesset Perturbation Theory (MP2), метод связанных кластеров (CCSD), взаимодействие конфигураций (QCISD). Использовалось такое равновесное межьядерное расстояние $R_{e}$, которое получалось оптимизацией в рамках использованного метода.

Во втором случае мы использовали для расчета одноконфигурационный конечно-разностный метод Хартри-Фока х2DHF [54], который дает слэтеровскую асимптотику радиальных волновых функций. Для величины $R_{e}$ принималось справочное значение [55].

Вычисления проводились в системе центра заряда ядер. По поводу другого выбора начала координат, что важно для расчетов дипольных моментов систем, обладающих избыточным зарядом, см. [56-58]. Ось $z$ выбиралась направленной от О к Н. Тогда при заданной электронной плотности $\rho_{e}(\mathbf{r})$ для дипольного и квадрупольного моментов имеем соответственно

$$
\begin{gathered}
d=-\int z \rho_{e}(\mathbf{r}) \mathrm{d}^{3} r, \\
Q_{z z}=\frac{8}{9} R_{e}^{2}-\frac{1}{2} \int \rho_{e}(\mathbf{r})\left(3 z^{2}-r^{2}\right) \mathrm{d}^{3} r .
\end{gathered}
$$


Таблица 1. Равновесное межъядерное расстояние, постоянные дипольный $d$ и квадрупольный $Q_{z z}$ моменты и поляризуемости радикал-катиона $\mathrm{OH}^{+}$в системе центра заряда ядер. Ось $z$ направлена от О к H; значения NIST взяты из базы [59], остальные значения вычислены в данной работе (,д.р.“)

\begin{tabular}{c|l|c|c|c|c|c|c|c|c}
\hline \multirow{2}{*}{ № } & \multirow{2}{*}{ Метод / базис } & \multirow{2}{*}{$R_{e}, \AA$} & \multicolumn{2}{|c|}{$d$, а.u. } & \multicolumn{2}{|c|}{$Q_{z z}$, а.u. } & \multicolumn{3}{|c}{ Поляризуемость, а.u. } \\
\cline { 4 - 9 } & & & д.p. & NIST & д.p. & NIST & $\alpha_{\perp}$ & $\alpha_{\|}$ & $\bar{\alpha}$ \\
\hline 1 & $\begin{array}{l}\text { wB97X-D/ } \\
\text { d-aug-cc-pVQZ }\end{array}$ & 1.0289 & 0.7904 & & 1.3405 & & 3.4563 & 4.9698 & 3.9608 \\
\hline 2 & $\begin{array}{l}\text { B97D3/ } \\
\text { Def2TZVPP }\end{array}$ & 1.0292 & 0.7954 & 0.784 & 1.3351 & 1.342 & 2.9949 & 4.9224 & $3.68[59]$ \\
\hline 3 & $\begin{array}{l}\text { QCISD/ } \\
\text { 6-31G(2df,p) }\end{array}$ & 1.0294 & 0.8410 & 0.841 & 1.3660 & & 3.0566 & 4.7472 & 3.6201 \\
\hline 4 & $\begin{array}{l}\text { CCSD/ } \\
\text { aug-cc-pVTZ }\end{array}$ & 1.0290 & 0.8338 & 0.792 & 1.3507 & 1.334 & 3.4265 & 5.0139 & 3.9556 \\
\hline 5 & $\begin{array}{l}\text { CCSD/ } \\
\text { d-aug-cc-pVTZ }\end{array}$ & 1.0291 & 0.8331 & 0.792 & 1.3526 & 1.335 & 3.4191 & 5.0041 & 3.9474 \\
\hline 6 & $\begin{array}{l}\text { MP2/ } \\
\text { d-aug-cc-pVDZ }\end{array}$ & 1.0276 & 0.8299 & & 1.3435 & 1.326 & 3.4530 & 4.9146 & $3.94[59]$ \\
\hline 7 & $\begin{array}{l}\text { RCCSD/ } \\
\text { aug-cc-pVTZ }\end{array}$ & 1.0289 & 0.8343 & & 1.3837 & & 3.4558 & 5.0352 & 3.9823 \\
\hline 8 & $\begin{array}{l}\text { RCCSD/ } \\
\text { d-aug-cc-pVTZ }\end{array}$ & 1.0290 & 0.8336 & & 1.3853 & & 3.4470 & 5.0254 & 3.9731 \\
\hline 9 & $\begin{array}{l}\text { CCSD+T(CCSD)/ } \\
\text { aug-cc-pVTZ }\end{array}$ & 1.0277 & 0.7912 & & & 1.319 & 3.4414 & 4.9984 & 3.9604 \\
\hline 10 & $\begin{array}{l}\text { CCSD+T(CCSD)/ } \\
\text { d-aug-cc-pVTZ }\end{array}$ & 1.0285 & 0.7915 & & & 1.322 & 3.4400 & 4.9862 & 3.9554 \\
\hline 11 & MCSCF [60] & 1.031 & 0.913 & & & & & & \\
\hline 12 & x2DHF & $1.029[54]$ & 0.8394 & & 1.4336 & & & &
\end{tabular}

Первое слагаемое в $Q_{z z}$ обусловлено вкладом положительных зарядов ядер. Второе слагаемое определяется электронной конфигурацией. Мы рассматриваем основной электронный терм $X^{3} \Sigma^{-}$с конфигурацией $1 \sigma^{2} 2 \sigma^{2} 3 \sigma^{2} 1 \pi_{+1} 1 \pi_{-1}$, где $\pi$-электроны не спарены и образуют спиновый триплет.

Статическая поляризуемость вычисляется здесь численным дифференцированием наведенного дипольного момента по напряженности внешнего поля.

Результаты расчетов даны в табл. 1. Для квантовохимических расчетов были использованы такие методы/базисы, которые для дипольного момента нейтрального радикала $\mathrm{OH}$ давали отличие от экспериментального значения $d(\mathrm{OH})=0.6531$ a.u., рекомендуемого NIST [59], менее $1 \%$. Для моментов $d$ и $Q_{z z}$ в ионе $\mathrm{OH}^{+}$ в строках 1-6 приведены результаты расчета с методами/базисами, дающими равновесное межьядерное расстояние $R_{e}$, отличающееся от табличного $R_{e}=1.029 \AA$ в пределах $0.1 \%$. Результаты в строках $1-5$ получены с использованием пакета MOLPRO, а в строке 6 с методом MP2 - с помощью пакета Gaussian. Методы
3-6 относят к классу пост-хартри-фоковских. Методы связанных кластеров CCSD и взаимодействия конфигураций QCISD являются непертурбативными многоконфигурационными.

Мы также исследовали дополнительно методы RCCSD, предотвращающие „спиновую контаминацию“ в системах с незамкнутыми оболочками. Как показано в строках 7,8 , это приводит к изменению дипольного момента менее, чем на $0.1 \%$ и квадрупольного момента на $\sim 1 \%$.

Методы DFT уменьшают время расчета на 2-3 порядка и дают для $R_{e}$ сопоставимые по точности $(<0.1 \%)$ с $\operatorname{RCCSD}(\mathrm{T})$ результаты.

Пертурбативный учет тройных возбуждений электронов методами $\operatorname{CCSD}(\mathrm{T})$ и $\mathrm{QCISD}(\mathrm{T})$ привел к изменению равновесного межъядерного расстояния на несколько процентов. Причиной такого расхождения может быть специфика использования данных методов (см. ниже).

Другой многоконфигурационный метод учета тройных возбуждений CCSD+T(CCSD), выполненный с использованием показавшего свою эффективность базисного 
набора aug-cc-pVTZ в пакете NWChem, дал приемлемые значения и для межъядерных расстояний $R_{e}$ в $\mathrm{OH}$ и $\mathrm{OH}^{+}$, и для дипольного момента ОН. Однако дипольный момент радикал-катиона $\mathrm{OH}^{+}$(строки 9, 10 в табл. 1) оказывается меньшим и сопоставимым с полученным методами функционала плотности.

Мы также протестировали возможности многочастичной теории возмущений 4-го порядка МВРТ4, реализованной в пакете NWChem. Несмотря на хорошее воспроизведение межъядерного расстояния, дипольный момент нейтрального радикала $\mathrm{OH}$ оказался отличающимся от табличного значения на $\sim 10$ \% при использовании корреляционно-согласованных базисных наборов. Поэтому мы не использовали метод МВРТ4 для расчета мультипольных моментов радикал-катиона $\mathrm{OH}^{+}$.

Для сравнения приведены также значения $R_{e}$ и $d$, полученные в работе [61] многоконфигурационным методом самосогласованного поля MCSCF в подходе Рутана-Хартри-Фока [60] (строка 11 в табл. 1). В цитированной работе были задействованы 7 конфигураций, которые в декартовых координатах имеют вид: $3 \sigma^{2} 1 \pi_{x} 1 \pi_{y}, 4 \sigma^{2} 1 \pi_{x} 1 \pi_{y}, 3 \sigma^{2}\left\{1 \pi_{x} 2 \pi_{y}-1 \pi_{y} 2 \pi_{x}\right\}$, $3 \sigma^{2} \quad 2 \pi_{x} \quad 2 \pi_{y}, \quad{ }^{1}\left(\begin{array}{lll}3 \sigma & 4 \sigma\end{array}\right) \quad\left\{\begin{array}{llll}1 \pi_{x} & 2 \pi_{y}-1 \pi_{y} & 2 \pi_{x}\end{array}\right\}$, ${ }^{1}(3 \sigma 4 \sigma) 1 \pi_{x} 1 \pi_{y},{ }^{3}(3 \sigma 4 \sigma)^{3}\left(1 \pi_{x} 1 \pi_{y}\right)$.

В строке 12 табл. 1 приведены данные, полученные конечно-разностным методом Хартри-Фока в сфероидальных координатах, предложенным в работе [54].

К сожалению, дипольный и квадрупольный моменты, найденные различными пост-хартри-фоковскими методами: связанных кластеров CCSD, взаимодействия конфигураций QCISD и Меллера-Плессета MP2 (строки 3-6 табл. 1), с базисными наборами, использованными в [59] для ОН, имеют достаточно большой разброс значений $\left(d=0.834 \pm 0.004, Q_{z z}=1.364 \pm 0.016\right)$.

Тем не менее упомянутый выше метод связанных кластеров признается одним из наиболее точных подходов современной квантовой химии. Точность расчета большинства молекулярных параметров, в частности, поляризуемостей, сравнима с точностью экспериментальных методов [62]. Однако корректный учет корреляционной энергии не может быть проведен даже методом $\operatorname{CCSD}(\mathrm{T})$ в базисах малого и среднего размера. По этой причине использование CCSD(T)/aug-cc-pVTZ ухудшало результат для равновесного межьядерного расстояния по сравнению с CCSD/aug-cc-pVTZ.

В табл. 2 приведены равновесные межъядерные расстояния, мультипольные моменты и статическая поляризуемость радикал-катиона $\mathrm{OH}^{+}$, вычисленные по теории функционала плотности wB97X-D и методом RCCSD(T) с различными базисными наборами x-aug-cc-pVXZ.

Метод RCCSD демонстрирует хорошую сходимость $R_{e}, d$ и $Q_{z z}$ радикал-катиона $\mathrm{OH}^{+}$с ростом параметра качества X в базисном наборе X-aug-cc-pVXZ. Существенного различия между *-pV5Z и *-pV6Z нет. Для радикал-катиона $\mathrm{OH}^{+}$поляризуемость практически не чувствительна к количеству диффузных функций х
Таблица 2. Равновесное межъядерное расстояние (в $\AA$ ), постоянные дипольный $d$ и квадрупольный $Q_{z z}$ моменты радикалкатиона $\mathrm{OH}^{+}$в системе центра заряда ядер и статическая поляризуемость (все в атомных единицах); ось $z$ направлена от $\mathrm{O}$ к H; базисные наборы х-aug-cc-pVXZ

\begin{tabular}{c|c|c|c|c|c|c|c}
\hline $\mathrm{x}$ & $\mathrm{X}$ & $R_{e}$ & $d$ & $Q_{z z}$ & $\alpha_{\perp}$ & $\alpha_{\|}$ & $\bar{\alpha}=\frac{1}{3} \mathrm{Sp} \alpha$ \\
\hline \multicolumn{7}{c}{$\mathrm{wB} 97 \mathrm{X}-\mathrm{D}$} \\
\hline \multirow{2}{*}{1} & $\mathrm{~T}$ & 1.0299 & 0.7907 & 1.3381 & 3.4606 & 5.0069 & 3.9760 \\
\cline { 2 - 8 } & $\mathrm{Q}$ & 1.0289 & 0.7904 & 1.3405 & 3.4563 & 4.9698 & 3.9608 \\
\hline & 5 & 1.0286 & 0.7904 & 1.3391 & 3.4668 & 4.9735 & 3.9690 \\
\hline \multirow{2}{*}{2} & $\mathrm{~T}$ & 1.0300 & 0.7900 & 1.3415 & 3.4554 & 4.9911 & 3.9673 \\
\hline & $\mathrm{Q}$ & 1.0289 & 0.7904 & 1.3392 & 3.4551 & 4.9686 & 3.9596 \\
\hline & 5 & 1.0286 & 0.7903 & 1.3392 & 3.4669 & 4.9734 & 3.9691 \\
\hline & 6 & 1.0286 & 0.7900 & 1.3392 & 3.4622 & 4.9690 & 3.9645 \\
\hline \multirow{2}{*}{1} & $\mathrm{~T}$ & 1.0312 & 0.8366 & 1.3933 & 3.4558 & 5.0352 & 3.9823 \\
\cline { 2 - 8 } & $\mathrm{Q}$ & 1.0283 & 0.8329 & 1.3839 & 3.4328 & 4.9742 & 3.9466 \\
\hline & 5 & 1.0280 & 0.8328 & 1.3824 & 3.4250 & 4.9572 & 3.9357 \\
\hline & 6 & 1.0280 & 0.8327 & 1.3824 & 3.4241 & 4.9537 & 3.9340 \\
\hline 2 & $\mathrm{~T}$ & 1.0313 & 0.8359 & 1.3949 & 3.4470 & 5.0254 & 3.9731 \\
\hline & $\mathrm{Q}$ & 1.0283 & 0.8329 & 1.3839 & 3.4285 & 4.9721 & 3.9430 \\
\hline & 5 & 1.0280 & 0.8328 & 1.3826 & 3.4247 & 4.9572 & 3.9355 \\
\hline 6 & 1.0280 & 0.8328 & 1.3824 & 3.4236 & 4.9539 & 3.9337 \\
\hline
\end{tabular}

на одну орбиталь. Различия отсутствуют уже при $\mathrm{x}=1$ (aug-*) или 2 (d-aug-*).

Для мультипольных моментов сходимость обоих методов тоже имеет место. Однако DFT дает заниженные значения по сравнению с $\operatorname{RCCSD}(\mathrm{T})$ на $5 \%$ для дипольных моментов и на $3 \%$ - для квадрупольных.

Для статических поляризуемостей результаты обоих методов сопоставимы. Однако в отличие от $\operatorname{RCCSD}(\mathrm{T})$ сходимость результатов DFT с ростом X оказывается более медленной и немонотонной.

Равновесное межъядерное расстояние $R_{e}=1.0280 \AA$ отличается от предложенного NIST [59] менее, чем на $0.1 \%$. Важность точного воспроизведения параметра $R_{e}$ показана в табл. 2 для расчета мультипольных моментов как функций межъядерного расстояния методом RCCSD(T)/aug-cc-pV5Z. Легко заметить, что изменение межъядерного расстояния в окрестности равновесного значения на $10 \%$ приводит к изменению дипольного момента на величину того же порядка, а квадрупольного момента - на $\sim 34 \%$. Такая чувствительность моментов 
Таблица 3. Функции дипольного $d$ и квадрупольного $Q_{z z}$ моментов радикал-катиона $\mathrm{OH}^{+}$в системе центра заряда ядер, найденные в расчетах $\operatorname{CCSD}(\mathrm{T}) /$ aug-cc-pV5Z; ось $z$ направлена от О к $\mathrm{H}$

\begin{tabular}{c|c|c}
\hline$R, \AA$ & $d$, a.u. & $Q_{z z}$, a.u. \\
\hline 0.9252 & 0.7326 & 0.9804 \\
\hline 0.9509 & 0.7552 & 1.0736 \\
\hline 0.9766 & 0.7787 & 1.1712 \\
\hline 1.0023 & 0.8031 & 1.2729 \\
\hline 1.0280 & 0.8328 & 1.3824 \\
\hline 1.0537 & 0.8548 & 1.4892 \\
\hline 1.0794 & 0.8820 & 1.6040 \\
\hline 1.1051 & 0.9102 & 1.7231 \\
\hline 1.1308 & 0.9394 & 1.8468
\end{tabular}

к выбору межъядерного расстояния отмечалась в работе [63].

Таким образом, для вычисления параметров $\mathrm{OH}^{+}$ там, где не оговорено особо, достаточно ограничиться RCCSD(T)/aug-cc-pV5Z. Значения мультипольных моментов, $d=0.833, Q_{z z}=1.382$, будут использованы в дальнейшем для расчета параметров ридберговских состояний радикала $\mathrm{OH}$.

\section{Ридберговские спектры}

\section{Область ВОА}

В этом и следующем разделах мы рассмотрим вклад дипольного момента молекулярного остова в ридберговские спектры радикала ОН. Состояния RE принято делить на проникающие и непроникающие. Для первых существенно короткодействующее взаимодействие RE с остовом катиона, тогда как для вторых это взаимодействие мало. Из литературы известно (см., например, $[25,64])$, что для двухатомных молекул непроникающими можно считать состояния RE с орбитальными моментами $l \geq 3$. В пределе ВОА это означает, что использование уравнений (2) для расчета спектров RE при $|m| \geq 3$ является вполне оправданным. Заметим, что описание проникающих состояний проводилось в некоторых работах в рамках довольно простых моделей (для атомов, например, см. [65]).

В табл. 4 приведены значения параметров ридберговских состояний радикала ОН в пределе ВОА. Проекции из интервала $0 \leq|m| \leq 2$ соответствуют проникающим состояниям. Для возможного сопоставления параметров $\mathrm{RE}$ с этими значениями $|m|$ с экспериментом необходимо учитывать короткодействующее взаимодействие RE c остовом. Как видно, для состояния с $m=0$ эффективный орбитальный момент $\tilde{L}$, соответствующий $L=0$, является комплексным. Это связано, как говорилось выше, с нарушением приближения точечного диполя. Для непроникающих состояний мы приводим результаты лишь для одного значения $|m|=3$, поскольку видно, что уже в этом случае отличие эффективных орбитального момента $\tilde{L}$ и главного квантового числа $v$ от целочисленных значений является малым. Это же заключение применимо и к вкладу в главное квантовое число $v$ квадрупольного момента. Для молекулы SO этот вклад учитывался в работе [44] и оказался мал в указанном выше смысле.

\section{Область IBOA}

Перейдем теперь к анализу области IBOA. В табл. 5 приведены параметры ридберговских состояний, аналогичные тем, что даны в табл. 4. Поскольку основным состоянием катиона $\mathrm{OH}^{+}$является триплет, проекция полного углового момента на ось молекулы $\omega \geq 1$. Как видно, наиболее сильно дипольный момент остова влияет на состояния $L=0$, что вполне естественно. В случае $\omega=j=J=2$ для этого состояния возникает даже „Падение на центр“, связанное с комплексностью эффективного орбитального момента $\tilde{L}$ и неприменимостью приближения точечного диполя. Обратим внимание на отрицательность $\tilde{L}$ для $L=0$ при других значениях параметров $\omega, j, J$. Это связано с тем, что при $L=0$ центробежное отталкивание RE от остова практически отсутствует, и дипольный момент притягивает RE к остову.

Таблица 4. Параметры ридберговских состояний радикала ОН в пределе ВОА, квантовохимический расчет статической поляризуемости $\mathrm{OH}^{+}$выполнен методом $\operatorname{RCCSD}(\mathrm{T}) /$ aug-cc-pV6Z

\begin{tabular}{r|c|c|r|r|c}
\hline$|m|$ & $L$ & $\tilde{L}$ & $\mu_{d}=L-\tilde{L}$ & $\mu_{Q}$ & $\mu_{\mathrm{pol}}$ \\
\hline \multirow{3}{*}{0} & 0 & $-0.5+0.392941 \mathrm{i}$ & - & - & - \\
& 1 & 1.071 & -0.071 & 0.184 & 0.678 \\
& 2 & 2.014 & -0.014 & 0.026 & 0.030 \\
\hline \multirow{3}{*}{1} & 1 & 0.954 & 0.046 & -0.092 & -0.064 \\
& 2 & 2.006 & -0.006 & 0.013 & 0.030 \\
& 3 & 3.003 & -0.003 & 0.007 & 0.005 \\
\hline \multirow{3}{*}{2} & 2 & 1.987 & 0.013 & -0.026 & -0.028 \\
& 3 & 3.000 & 0.000 & 0.000 & 0.005 \\
& 4 & 4.001 & -0.001 & 0.002 & 0.001 \\
\hline \multirow{3}{*}{3} & 3 & 2.994 & 0.006 & -0.011 & -0.004 \\
& 4 & 3.999 & 0.001 & -0.001 & -0.001 \\
& 5 & 5.000 & 0.000 & 0.000 & 0.000
\end{tabular}


Таблица 5. Параметры ридберговских состояний радикала ОН в пределе IBOA

\begin{tabular}{|c|c|c|c|c|}
\hline$\omega$ & $j$ & $J$ & $L$ & $\tilde{L}$ \\
\hline 1 & 1 & 1 & $\begin{array}{l}0 \\
1 \\
2\end{array}$ & $\begin{array}{c}-0.307 \\
1.051 \\
2.012\end{array}$ \\
\hline 1 & 1 & 2 & $\begin{array}{l}1 \\
2 \\
3\end{array}$ & $\begin{array}{l}0.966 \\
2.011 \\
3.007\end{array}$ \\
\hline 1 & 1 & 3 & $\begin{array}{l}2 \\
3 \\
4\end{array}$ & $\begin{array}{l}1.987 \\
3.004 \\
4.004\end{array}$ \\
\hline 1 & 2 & 1 & $\begin{array}{l}1 \\
2 \\
3\end{array}$ & $\begin{array}{l}0.996 \\
2.001 \\
3.001\end{array}$ \\
\hline 1 & 2 & 2 & $\begin{array}{l}0 \\
1 \\
2 \\
3 \\
4\end{array}$ & $\begin{array}{c}-0.082 \\
1.016 \\
2.003 \\
3.001 \\
4.001\end{array}$ \\
\hline 1 & 2 & 3 & $\begin{array}{l}1 \\
2 \\
3 \\
4 \\
5\end{array}$ & $\begin{array}{l}0.990 \\
2.002 \\
3.001 \\
4.001 \\
5.000\end{array}$ \\
\hline 2 & 2 & 1 & $\begin{array}{l}1 \\
2 \\
3\end{array}$ & $\begin{array}{l}0.984 \\
2.005 \\
3.003\end{array}$ \\
\hline 2 & 2 & 2 & $\begin{array}{l}0 \\
1 \\
2 \\
3 \\
4\end{array}$ & $\begin{array}{c}-0.5+0.171 \mathrm{i} \\
1.056 \\
2.011 \\
3.004 \\
4.002\end{array}$ \\
\hline 2 & 2 & 3 & $\begin{array}{l}1 \\
2 \\
3 \\
4 \\
5\end{array}$ & $\begin{array}{l}0.959 \\
2.008 \\
3.005 \\
4.003 \\
5.002\end{array}$ \\
\hline
\end{tabular}

\section{Зависимость энергии молекулы от полного углового момента}

На рисунке показаны зависимости энергий RE, определяемых по формуле (10), от полного момента молекулы $J$ для значений $\omega=1,2 ; j=2, L=1$. Численные данные взяты из табл. 5. Как видно, эти зависимости принципиально отличаются от зависимостей типа $\sim J(J+1)$, характерных для энергий ротатора. К сожалению, получение значений вращательных энергий для бо́льших значений $J$ не представляется возможным из-за малого отличия спектра RE от чисто кулоновского спектра.

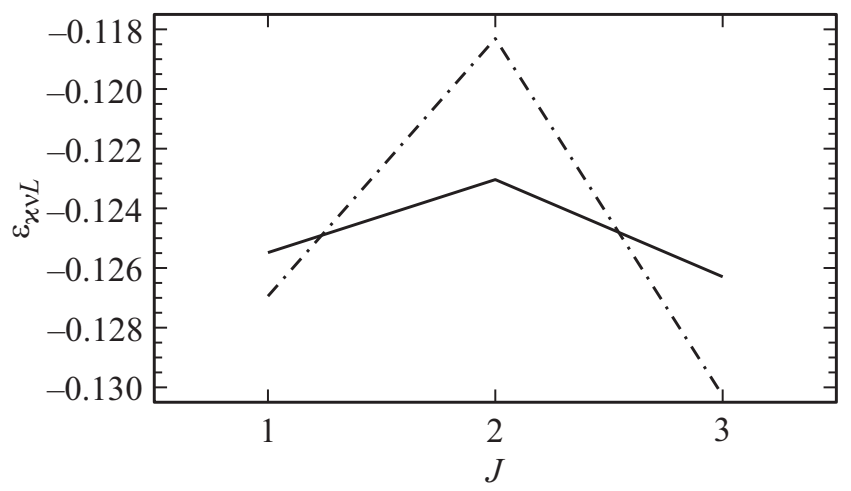

Зависимость энергии RE $\varepsilon_{\mathcal{x v L}}$ от полного момента молекулы $J$. Сплошная линия: $\omega=1$, штрихпунктирная: $\omega=2$. В обоих случаях $j=2, L=1$.

\section{Заключение}

Проведенные расчеты показывают, что для молекул с величиной дипольного момента такого порядка, как у радикала $\mathrm{OH}, d \simeq 0.8$ a.u., перемешивание состояний ридберговского электрона с разными орбитальными моментами относительно мало́. Исключение в области применимости приближения Борна-Оппенгеймера составляют состояния с $m=L=0$, для которых, однако, неприменимо приближение точечного диполя. Аналогичный вывод следует и для состояний $\omega=j=J=2$, $L=0$ в области обратного приближения Борна-Оппенгеймера. Проведенные расчеты вращательных энергий ридберговских состояний ОН показывают их принципиальное отличие от спектров квантового ротатора $\sim J(J+1)$.

\section{Благодарности}

Авторы благодарят Воронежский госуниверситет за предоставленный высокопроизводительный компьютерный центр параллельных вычислений для проведения расчетов.

\section{Финансирование работы}

Работа выполнена при финансовой поддержке РФФИ (проект 19-52-26006) и Чешского научного фонда (GAČR, проект 20-10591J), а также ERDF/ESF „Centre of Advanced Applied Sciences“ (проект CZ.02.1.01/0.0/0.0/16_019/0000778).

\section{Конфликт интересов}

Авторы заявляют, что у них нет конфликта интересов.

\section{Список литературы}

[1] Зверева Г.Н. // Опт. и спектр. 2010. Т. 108. С. 963-970; Zvereva G.N. // Opt. Spectrosc. 2010. V. 108. N 6. P. 915-922. doi 10.1134/S0030400X10060135 
[2] Корбут А.Н., Кельман В.А., Жменяк Ю.В., Кленовский М.С. // Опт. и спектр. 2014. Т. 116. С. 995-1002; Korbut A.N., Kelman V.A., Zhmenyak Yu.V., Klenovskii M.S. // Opt. Spectrosc. 2014. V. 116. N 6. P 919-925. doi 10.1134/S0030400X14040146

[3] Шуаибов А.К., Миня А.И., Гомоки 3.Т., Грицак Р.В. // Опт. и спектр. 2013. Т. 114. С. 212-215; Shuaibov A.K., Minya A.I., Gomoki Z.T., Gritsak R.V. // Opt. Spectrosc. 2013. V. 114. N 2. P. 193-196. doi 10.1134/S0030400X13020264

[4] Беловолова Л.В. // Опт. и спектр. 2020. Т. 128. С. 923-942; Belovolova L.V. // Opt. Spectrosc. 2020. V. 128. N 7. P 932-951. doi 10.21883/OS.2020.07.49565.64-20

[5] Wöhler C., Grumpe A., Berezhnoy A.A., Shevchenko V.V. // Sci. Adv. 2017. V. 3. N 9. P. e1701286. doi 10.1126/sciadv.1701286

[6] Wöhler C., Grumpe A., Bhatt M., Berezhnoy A.A. et al. // Astron. \& Astrophys. 2019. V. 630. P. L7. doi 10.1051/00046361/201935927

[7] Valiev R.R., Berezhnoy A.A., Sidorenko A.D., Merzlikin B.S., Cherepanov V.N. // Phys. Sol. State. 2017. V. 145. P. 38-48. doi 10.1016/j.pss.2017.07.011

[8] Grumpe A., Wöhler C., Berezhnoy A.A., Shevchenko V.V. // Icarus. 2019. V. 321. P. 486-507. doi https://doi.prg/10.1016/j.icarus.2018.11.025

[9] Valiev R.R., Berezhnoy A.A., Gritsenko I.S., Merzlikin B.S. et al. // Astron. \& Astrophys. 2020. V. 633. P. A39. doi 10.1051/0004-6361/201936230

[10] Shelkovnikov A., Butcher R.J., Chardonnet C., AmyKlein A. // Phys. Rev. Lett. 2008. V. 100. P. 150801. doi 10.1103/PhysRevLett.100.150801

[11] Kozlov M.G. // Phys. Rev. A. 2009. V. 80. P. 022118. doi 10.1103/PhysRevA.80.022118

[12] Flambaum V.V., Wiringa R.B. // Phys. Rev. C. 2009. V. 79. P. 034302. doi 10.1103/PhysRevC.79.034302

[13] Kanekar N., Langston G.I., Stocke J.T., Carilli C.L., Menten K.M. // Astrophys. J. Lett. 2012. V. 746. N 2. P. L16. doi 10.1088/2041-8205/746/2/116

[14] Wall T.E. // J. Phys. B: At. Mol. Opt. Phys. 2016. V. 49. N 24. P. 243001. doi 10.1088/0953-4075/49/24/243001

[15] Safronova M.S., Budker D., DeMille D., Kimball D.F.J. et al. // Rev. Mod. Phys. 2018. V. 90. P. 025008. doi 10.1103/RevModPhys.90.025008

[16] Seaton M.J. // Proc. Phys. Soc. (London). 1966. V. 88. N 4. P. 801-814. doi 10.1088/0370-1328/88/4/302

[17] Seaton M.J. // Proc. Phys. Soc. (London). 1966. V. 88. N 4. P. $815-832$. doi $10.1088 / 0370-1328 / 88 / 4 / 303$

[18] Fano U. // Phys. Rev. A. 1970. V. 2. P. 353-365. doi 10.1103/PhysRevA.2.353

[19] Greene C., Fano U., Strinati G. // Phys. Rev. A. 1979. V. 19. P. 1485-1509. doi 10.1103/PhysRevA.19.1485

[20] Greene C.H. // Phys. Rev. A. 1979. V. 20. P. 656-669. doi 10.1103/PhysRevA.20.656

[21] Jungen C., Atabek O. // J. Chem. Phys. 1977. V. 66. N 12. P. 5584-5609. doi 10.1063/1.433881

[22] Jungen C., Dill D. // J. Chem. Phys. 1980. V. 73. N 7. P. 3338-3345. doi 10.1063/1.440528

[23] Jungen C., Miescher E. // Can. J. Phys. 1969. V. 47. N 17. P. 1769-1787. doi 10.1139/p69-225

[24] Murphy J.E., Friedman-Hill E., Field R.W. // J. Chem. Phys. 1995. V. 103. N 15. P. 6459-6466. doi 10.1063/1.470423

[25] Arif M., Jungen C., Roche A.L. // J. Chem. Phys. 1997. V. 106. N 10. P. 4102-4118. doi 10.1063/1.473124
[26] Jungen C., Roche A.L. // Can. J. Phys. 2001. V. 79. N 2-3. P. 287-298. doi 10.1139/p00-099

[27] Altunata S.N., Coy S.L., Field R.W. // J. Chem. Phys. 2005. V. 123. N 8. P. 084319. doi 10.1063/1.2005041

[28] Altunata S.N., Coy S.L., Field R.W. // J. Chem. Phys. 2006. V. 124. N 19. P. 194302 . doi $10.1063 / 1.2192518$

[29] Kay J.J., Coy S.L., Wong B.M., Jungen C., Field R.W. // J. Chem. Phys. 2011. V. 134. N 11. P. 114313. doi 10.1063/1.3565967

[30] Jakubek Z.J., Field R.W. // Phys. Rev. Lett. 1994. V. 72. P. 2167-2170. doi 10.1103/PhysRevLett.72.2167

[31] Jakubek Z.J., Field R.W. // J. Mol. Spectrosc. 1996. V. 179. P. 99-124. doi 10.1006/jmsp.1996.0189

[32] Jakubek Z.J., Field R.W. // Philos. Trans. R. Soc. London, Ser. A. 1997. V. 355. N 1729. P. 1507-1526. doi 10.1098/rsta.1997.0073

[33] Jakubek Z.J., Field R.W. // J. Mol. Spectrosc. 2001. V. 205. N 2. P. 197 - 220. doi $10.1006 /$ jmsp. 2000.8264

[34] Raouafi S., Jeung G.-H., Jungen C. // J. Mol. Spectrosc. 1999. V. 196 . N 2. P. $248-258$. doi 10.1006/jmsp. 1999.7884

[35] Li J., Liu Y., Moss D.B., Gittins C.M. et al. // J. Mol. Spectrosc. 1999. V. 193. N 2. P. 403-411. doi 10.1006/jmsp.1998.7746

[36] Clevenger J. O., Harris N.A., Field R.W., Li J. // J. Mol. Spectrosc. 1999. V. 193. N 2. P. 412-417. doi 10.1006/jmsp.1998.7755

[37] Elfimov S.V., Dorofeev D.L., Zon B.A. // Phys. Rev. A. 2014. V. 89. P. 022507. doi 10.1103/PhysRevA.89.022507

[38] Зон Б.А. // ЖЭТФ. 1992. Т. 102. № 1. С. 36-46; Zon B.A. // JETP. 1992. V. 75. N 1. P. 36-46.

[39] Watson J. K.G. // Mol. Phys. 1994. V. 81. N 2. P. 277-289. doi 10.1080/00268979400100191

[40] Zon B.A. // Phys. Lett. A. 1995. V. 203. N 5-6. P. 373-375. doi 10.1016/0375-9601(95)00447-B

[41] Kozlov S.V., Pazyuk E.A., Stolyarov A.V. // Phys. Rev. A. 2016. V. 94. P. 042510. doi 10.1103/PhysRevA.94.042510

[42] Козлов Т.В., Пазюк Е.А., Столяров А.В. // Опт. и спектр. 2017. T. 123. C. 672-677; Kozlov S.V., Pazyuk E.A., Stolyarov A.V. // Opt. Spectrosc. 2017. V. 123. N 5. P 676-681. doi 10.7868/S0030403417110149

[43] Пазюк Е.А., Пупышев В.И., Зайцевский А.В., Столяров А.В. // Журн. физ. химии. 2019. Т. 93. С. 1461-1469; Pazyuk E.A., Pupyshev V.I., Zaitsevskii A.V., Stolyarov A.V. // Russ. J. Phys. Chem. 2019. V. 93. N 10. P 1865-1872.

[44] Dorofeev D.L., Elfimov S.V., Zon B.A. // Phys. Rev. A. 2012. V. 85. P. 022509. doi 10.1103/PhysRevA.85.022509

[45] Дорофеев Д.Л., Зон Б.А. // ЖЭТФ. 1996. Т. 110. № 3. C. 882-890; Dorofeev D.L., Zon B.A. // JETP. 1996. V. 86. N 3. P 485-489.

[46] Sun G., Zhou W., Zheng X., Qin Y. et al. // Mol. Phys. 2021. V. 119. N 1-2. P. e1837974. doi 10.1080/00268976.2020.1837974

[47] Червинская А.С., Елфимов Т.В., Дорофеев Д.Л., Чернов В.Е., Зон Б.А. // Опт. и спектр. 2017. Т. 122. C. 720-725; Chervinskaya A.S., Elfimov S.V., Dorofeev D.L., Chernov V.E., Zon B.A. // Opt. Spectrosc. 2017. V. 122. N 5. P. 699-704. doi 10.7868/S0030403417050051

[48] Ландау Л.Д., Лифиии Е.М. Квантовая механика (нерелятивистская теория). М.: ФИЗМАТЛИТ, 2002. 808 c.; Landau L.D., Lifshitz E.M. Quantum Mechanics, Nonrelativistic Theory. Oxford: Pergamon, 1991.

[49] Chernov V.E., Dolgikh A.V., Zon B.A. // Phys. Rev. A. 2005. V. 72. N 5. P. 052701. doi 10.1103/PhysRevA.72.052701 
[50] Данилян А.В., Чернов В.Е. // Опт. и спектр. 2008. Т. 104. № 1. C. 26-44; Danilyan A.V., Chernov V.E. // Opt. Spectrosc. 2008. V. 104. N 1. P 21-39. doi 10.1134/S0030400X08010050

[51] Frisch M.J. Gaussian 16 Revision A.02. 2016. Gaussian Inc. Wallingford CT.

[52] Werner H.-J., Knowles P.J., Manby F.R., Black J.A. et al. // J. Chem. Phys. 2020. V. 152. N 14. P. 144107. doi $10.1063 / 5.0005081$

[53] Valiev M., Bylaska E.J., Govind N., Kowalski K. et al. // Comput. Phys. Commun. 2010. V. 181. N 9. P. 1477-1489. doi 10.1016/j.cpc.2010.04.018

[54] Kobus J. // Comput. Phys. Commun. 2013. V. 184. N 3. P. 799-811. doi 10.1016/j.cpc.2012.09.033

[55] Радииг А.А., Смирнов Б.М. Параметры атомов и атомных ионов. М.: Энергоатомиздат, 1986. 344 с.; Radzig A.A., Smirnov B.M. Reference data on atoms, molecules and ions. Berlin: Springer-Verlag, 1985. 466 p. doi 10.1007/978-3-64282048-9

[56] Buckingham A.D., Longuet-Higgins H.C. // Mol. Phys. 1968. V. 14. N 1. P. 63-72. doi 10.1080/00268976800100051

[57] Gramada A., Bourne P.E. // Phys. Rev. E. 2008. V. 78. P. 066601. doi 10.1103/PhysRevE.78.066601

[58] Chervinskaya A.S., Dorofeev D.L., Elfimov S.V., Zon B.A. // Mol. Phys. 2020. V. 118. N 7. P. e1659433.

[59] NIST computational chemistry comparison and benchmark database (release 21). 2020. URL: http://cccbdb.nist.gov (online; accessed: August 2020)

[60] Werner H.-J., Rosmus P., Reinsch E.-A. // J. Chem. Phys. 1983. V. 79. N 2. P. 905-916. doi 10.1063/1.445867

[61] Adamowicz L. // J. Chem. Phys. 1988. V. 89. N 10. P. 6305-6309. doi 10.1063/1.455396

[62] Корнев А.С., Чернов В.Е., Зон Б.А. // Опт. и спектр. 2021. T. 129. C. 22-26; Kornev A.S., Chernov V.E., Zon B.A. // Opt. Spectrosc. 2021. V. 129. N 1. P 18-22. doi 10.21883/OS.2021.01.50434.224-20

[63] Watts J.D., Gauss J., Bartlett R.J. // Chem. Phys. Lett. 1992. V. 200. N 1. P. 1-7. doi 10.1016/0009-2614(92)87036-O

[64] Eyler E.E. // Phys. Rev. A. 1986. V. 34. P. 2881-2888. doi 10.1103/PhysRevA.34.2881

[65] Kornev A.S., Zon B.A. // J. Phys. B: At. Mol. Opt. Phys. 2003. V. 36. N 19. P. 4027-4034. doi 10.1088/0953-4075/36/19/011 